\title{
Categorical Management of Faculty in High Education: a case of University of Queensland
}

\author{
Sichao Chen ${ }^{1, a,{ }^{*}}$, Biao Ying ${ }^{1, b}$, Chunfeng Lv ${ }^{1, c}$, Qing Xu ${ }^{1, d}$ \\ ${ }^{1}$ Zhejiang University, Hangzhou, China \\ achensichao@zju.edu.cn, byingbiao@zju.edu.cn, `Ivchunfeng@zju.edu.cn, dxuqing@zju.edu.cn \\ ${ }^{*}$ Corresponding author
}

\begin{abstract}
This study introduced the detail of categorical management of faculty in the University of Queensland, which would provide potential suggestions for domestic universities in their reforms on faculty management. The results indicate: first, the University of Queensland adopts, the University of Queensland pay attention to both the processes and ultimate outcomes of faculties' work. On the contrast, domestic universities often only pay attention to the outcomes. Given these, we argue several important points on faculty evaluation: 1.The evaluation should be more concrete and targeted. 2. The evaluation should be as simple as possible to operate whether it's qualitative-oriented or qualitative-oriented. 3. To protect faculties' academic freedom on the basis of fairness. 4. All constructive academic efforts should be included not only explicit academic achievements, but also the tacit academic creation.
\end{abstract}

Keywords: Categorical Management of Faculty, Academic evaluation.

\section{国外教师分类管理制度介绍与分析一一以昆士兰大学为例}

\author{
陈思潮 $1, a,{ }^{*}$ ，应风䢠 $1, b$, 吕春风 ${ }^{1, c}$, 徐青 ${ }^{1, d}$ \\ 1浙江大学, 杭州, 浙江, 中国
}

achensichao@zju.edu.cn, byingbiao@zju.edu.cn, '9vchunfeng@zju.edu.cn, xuqing@zju.edu.cn *通讯作者

中文摘要. 本文通过对昆士兰大学教师分 类管理的介绍和分析, 探讨高校教师分类管 理问题, 为给国内高校教师分类改革提供意 见和建议。研究发现: 1. 昆士兰大学偏向定 性考核，国内高校则更偏向定量考核; 2 . 昆士兰大学兼顾教师工作过程与结果, 国内 高校更注重于工作结果。对此, 本文提出在 制度设置上需遵从以下几个原则: 1 . 有用 性, 评价不能泛泛而谈, 而要有明确的目的 性, 能促进学校和教师的共同发展; 2. 可操 作性, 无论以定性评价为主还是以定量评价 为主, 都应简便易行; 3. 在公平公正的基础
上, 充分保障教师的学术自由; 4. 全面性, 应包含所有富有建设性的学术努力, 不仅仅 重视显性的学术成就, 也应包括隐性的学术 创造。

\section{关键词：教师分类管理；学术评价;}

\section{1. 引言}

学术是大学的命脉和核心, 学术评价则 是大学发展的风向标。对何为学术的不同认 识, 对学术评价和教师奖励的不同制度设 计, 在很大程度上决定了教师的价值取向和 工作重点 ${ }^{[1]}$ 。根据学术评价对象的不同, 存 
在多种学术评价形式, 如学术机构评价、学 术人员评价、研究成果评价、研究项目评价、 研究活动评价等等。其中, 学术人员评价是 晋级、职称评聘、科学研究投入的重要依据。 高校教师学术评价属于学术人员评价范畴, 是指评价者在全面收集、整理、处理和分析 高校教师学术活动信息的基础上, 对其学术 工作成果进行事实判断和价值判断的过程 [2]。其功能主要体现在如下四个方面: 鉴定 学术人员、学术活动及学术成果的价值、认 可学术成就; 为学术决策提供依据; 为学术 活动提供规范; 促进学术研究质量的不断提 高 ${ }^{[3]}$ 。

随着教育改革的深化和发展, 我国高校 在提升人才培养水平、增强科学研究能力、 服务经济社会发展、推进文化传承创新方面 取得了较大成效。同时, 我国高校的学术评 价制度仍存在一系列问题, 主要包括: 制度 设计的不完善、过于注重量化考核、过于重 视科研忽视教学、矮化人文社科、学术腐败 道德失范等。针对学术评价制度, 学术界展 开了激烈的讨论, 讨论焦点集中在以下几个 方面: 定性评价和定量评价、个体评价和团 队评价、结果评价和过程评价等。面对这些 焦点问题, 我们认为首先应开展的是教师的 分类管理研究, 只有在分类的基础上, 才能 针对每一种类别的特征进行科学合理的评 价制度设计。

关于学术活动的分类, 教育界已经有了 不少的讨论。Boyer(1990)在《学术再思考》 中将教师的学术活动划分为四种相互独立 但又交叉的类型: 发现的学术、综合的学术、 应用的学术、教学的学术 ${ }^{[4]}$ 。其中, 发现的 学术和教学的学术被认为是学术活动的核 心, 即发现创造重要的新知识和在现有知识 领域培养学生, 并使其更进一步 ${ }^{[5]}$ 。有的学 者针对具体的教育组织研究教师学术活动 分类, Soleiman等人通过对伊朗40家医学院 的调查发现, 医学院教师的工作主要包含教 学、研究、临床医疗服务、管理、自我发展 五项 ${ }^{[6]}$ 。高校教师的工作, 确实不能狭险地 理解为 “教学” 和 “研究”, 还需要包括社 会服务、学院资金募集、研究经费募集、产 学合作、新人提携、学生就业和学校日常工 作等内容 ${ }^{[7]}$; Patricia和Michelle即将教师工
作划分为三类: 研究、教学和服务 ${ }^{[8]}$ 。这种 分类是学术界的主流观点, 基本涵盖了上述 几种分类的内容, 并且相对简单易于操作, 并在全球很多高校中得到应用。澳大利亚昆 士兰大学的教师分类采用的就是这种分类 框架, 本文将通过对昆士兰大学教师分类制 度的介绍, 为给国内高校教师分类改革提供 意见和建议。

\section{2. 昆士兰大学教师分类管理制度分析}

\section{1 案例介绍}

昆士兰大学坐落在澳大利亚昆士兰州 首府布里斯班市, 始建于1910年, 是澳大利 亚一所领先的教学和研究型综合性学府, 以 优美的校园环境、精良的教学设施和优秀的 师资队伍而斐声国际。昆士兰大学是澳大利 亚八大名校联盟（Group of Eight）、世界大 学联盟成员, 其科学研究的经费及学术水平 在澳大利亚的大学之中始终位居前三名, 2014年QS世界大学排名43位。经过一百多 年的建设, 昆士兰大学已经形成了成熟而规 范的教师学术评价体系, 在不同学科统一推 行。

\section{2 多通道的教师分类管理}

昆士兰大学的教师分类框架包括横向 与纵向两大结构。横向的分类是根据学校对 工作内容的认定, 将教师分为教学研究岗、 偏重教学岗、纯粹研究岗、应用研究岗四种 类型; 纵向的分类是根据教师的职业等级, 分为从 $\mathrm{A}$ 到 $\mathrm{E}$ 的 5 等级。

表1 昆士兰大学教师岗位分类定义表

\begin{tabular}{|c|c|}
\hline $\begin{array}{l}\text { 岗位名 } \\
\text { 称 }\end{array}$ & 角色定义 \\
\hline $\begin{array}{l}\text { 教学研 } \\
\text { 究岗 }\end{array}$ & $\begin{array}{l}\text { 主要学术活动是教学和研究, 社会服务同样被要求完成, } \\
\text { 教学学术是额外鼓励的。 }\end{array}$ \\
\hline 偏重教 & $\begin{array}{l}\text { 主要的工作是教学和教学学术, 促进学科知识传播与社会 } \\
\text { 服务同样被要求完成。 }\end{array}$ \\
\hline $\begin{array}{l}\text { 纯粹研 } \\
\text { 究岗 }\end{array}$ & $\begin{array}{l}\text { 主要工作是进行研究, 包括对研究生的管理。本科和研究 } \\
\text { 生教学以及社会服务同样被要求完成。 }\end{array}$ \\
\hline $\begin{array}{l}\text { 应用研 } \\
\text { 究岗 }\end{array}$ & $\begin{array}{l}\text { 主要工作是进行本科生、研究生和职业应用性的教学与研 } \\
\text { 究。参与应用性职业工作、并进行应用性创新以及传播创 } \\
\text { 新影响同样被要求。 }\end{array}$ \\
\hline
\end{tabular}

这种横向多通道岗位设置的分类方法 有助于：1.不同类型人才的特长发挥, 更加 公平公正地进行工作评价; 2. 学校构建一支 结构更为科学合理的教师队伍, 展开高效的 
人力资源管理；3.教师确定自我工作重点, 平衡各项工作之间的关系。

在纵向的分类方法中, 昆士兰大学将每 种教师横向类别都分为了从 $\mathrm{A}$ 到 $\mathrm{E}$ 五个等 级, 其中 $\mathrm{E}$ 为最高等级。每种等级下都设置 了具体的工作指标, 当满足上一级指标时, 教师便可申请升级, 如要续任, 则需要满足 其所在级别的工作指标要求。这种纵向分类 的制度设计有助于: 1 . 横向分类中不同类型 教师明确自身工作努力方向, 规划各自职业 生涯渠道中的发展计划; 2. 教师产权边界和 对组织的贡献明晰化; 3. 开展教师工资薪酬 的分类设置, 对教师队伍进行阶梯化管理; 4. 对教师资源的合理利用, 鼓励能力更强的 教师从事产出更多、更为重要的工作。

\section{3 学术活动考核指标}

如前所述, 昆士兰大学对教师学术活动 的考核, 采用了Patricia和Michelle的分类框 架, 即从研究、教学和服务三个方面对教师 的学术活动进行考核。

\subsection{1 研究}

昆士兰大学将研究工作界定为: 1 . 进行 满足DEEWR (The Department of Education, Employment and Workplace Relations) 标 准的研究活动。这里是指广义的研究, 包括 研究和研发, 指在现有知识系统基础上进行 的创造性工作和将现有知识进行应用化; 2 . 进行支持研究或符合研究定义的活动, 例如 管理参与研究人员或为他们提供专业、技 术、文书帮助和管理研究生研究; 3. 参与技 术转让和商业化。很有意思的是第二点, 昆 士兰大学将有助于研究活动所进行的辅助 活动 (更类似于校内服务工作) 也纳入研究 活动范围。

在学术研究活动方面, 昆士兰大学进一 步设置了详细的考核指标体系, 包括三大方 面37项评估指标: 活动参与度指标 (14项) 、 活动质量指标 (14项) 和活动影响力指标 (9 项），具体的指标如表2所示。
表2 昆士兰大学的研究工作评估指标

\begin{tabular}{|c|c|c|}
\hline $\begin{array}{c}\text { 活动参与度指 } \\
\text { 标 }\end{array}$ & 活动质量指标 & 活动影响力指标 \\
\hline 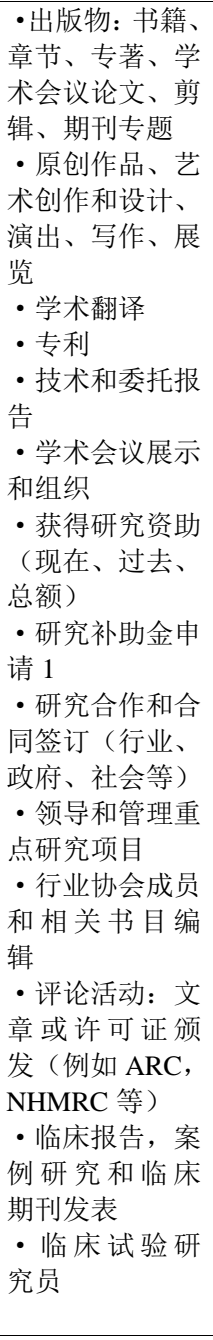 & 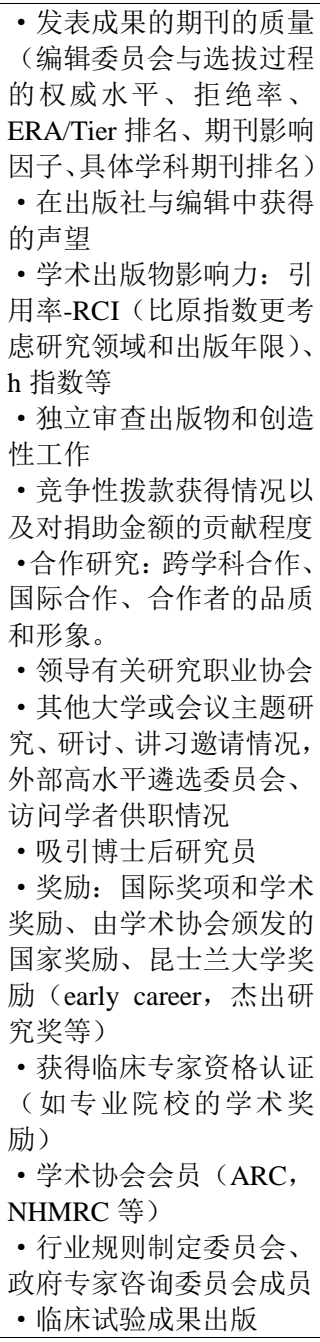 & $\begin{array}{l}\text { •研究商业化程度: } \\
\text { 专利、专利许可证、 } \\
\text { 衍生公司、技术转 } \\
\text { 移成功案例) } \\
\text {-与行业、政府、 } \\
\text { 公司、非盈利组织、 } \\
\text { 社区组织之间的研 } \\
\text { 究合作 } \\
\text { - 研究成果影响社 } \\
\text { 会或政府政策制定 } \\
\text { - 奖励: 行业、政 } \\
\text { 府和社会奖励、来 } \\
\text { 自行业协会的国家 } \\
\text { 级奖励 } \\
\text { - 衍生到最终用户 } \\
\text { 的研究, 包括社会、 } \\
\text { 行业和 政府群体 } \\
\text { (例如媒体评论、 } \\
\text { 系列讲座、宣传活 } \\
\text { 动等) } \\
\text { - 为社会、行业、 } \\
\text { 政府或其他群体提 } \\
\text { 供专家建议、技术 } \\
\text { 报告和咨询服务 } \\
\text { - 政府专家咨询委 } \\
\text { 员会、专家工作小 } \\
\text { 组成员 } \\
\text { - 受邀成为有关研 } \\
\text { 究领域的比赛活动商业果投等带来的影响 } \\
\text { 评审 } \\
\text { 资床 }\end{array}$ \\
\hline
\end{tabular}

\section{3 .2 教学}

对教学工作的考核, 昆士兰大学是从两 个与教学相关的教师活动——教学和教学 学术进行评估的。其中, 对教学活动的考核 指标包括: 熟悉学科领域最新发展; 了解学 科领域最新教学思想; 承担 (或协作)一系 列该学科领域课程; 指导学生完成研究和专 业项目; 保持改进提升课程设计、教学资源 和教学方法、采用该学科领域的新思想与新 教学方式。对教学学术活动的考核指标包 括: 通过发展、实践、评价、沟通等手段改 进教学方法、学习过程、课程、政策和教材 的设计; 理解掌握人类学习方式、促进更高 效学习的方法以及这些方法在特定学科背 景下的不同设置; 将各种思想 (包括新突破 和创新、可以被复制和阐述的、可以被文本 化以接受同行审议）应用于实践。 
对教学活动和教学学术活动的考核指 标体系, 同样设置了活动参与度指标、活动 质量和影响力指标, 共有28项教学活动评估 指标、20项教学学术评估指标, 具体的指标 如表3所示。

表3 昆士兰大学的教学工作评估指标

\begin{tabular}{|c|c|}
\hline 活动参与度指标 & 活动质量和影响力指标 \\
\hline 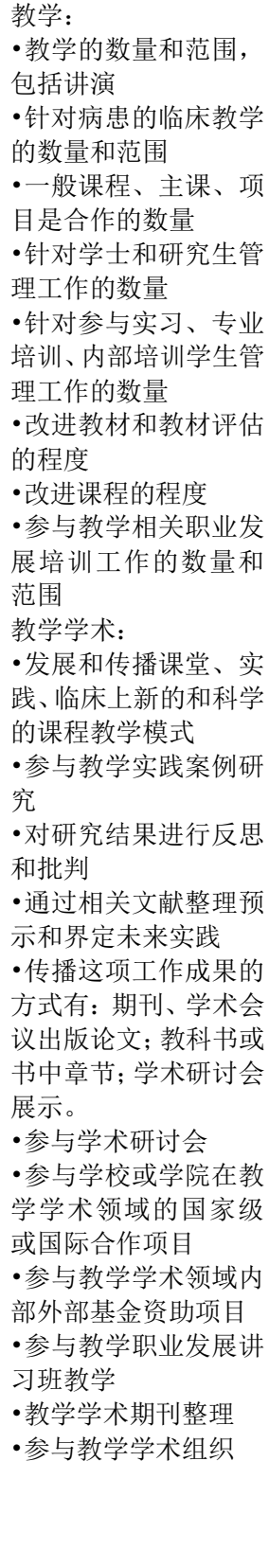 & 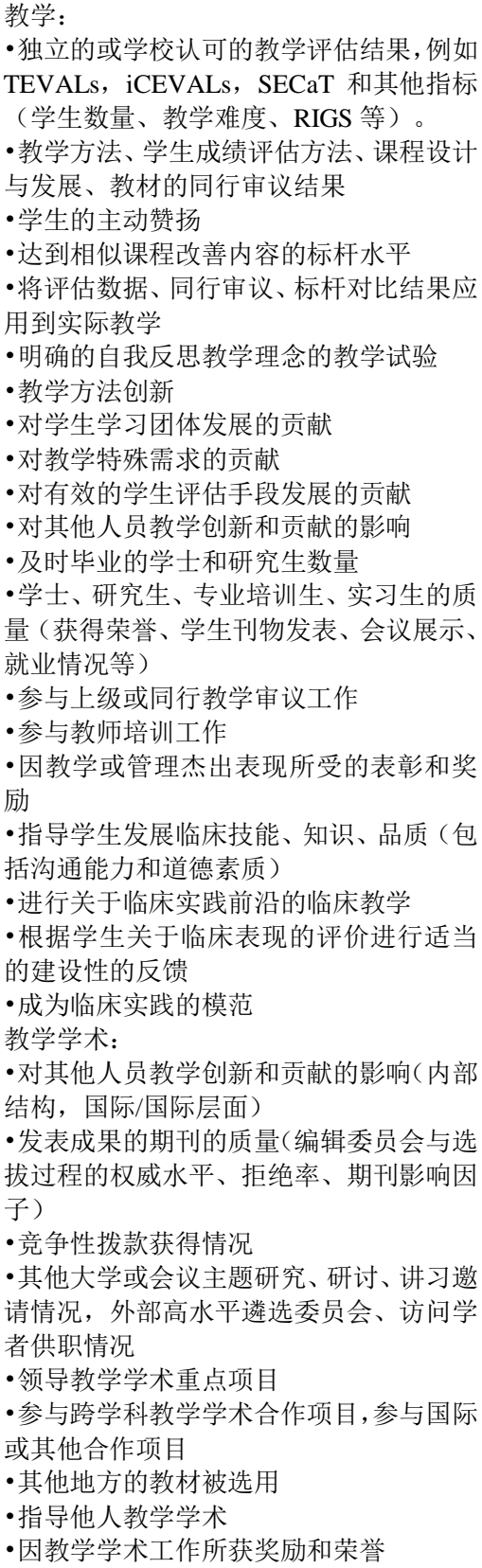 \\
\hline
\end{tabular}

注: 昆士兰大学是综合性大学, 包括医学院, 故对教学活动的评估里包含了临床教学的 评估指标。

\subsection{3 服务}

昆士兰大学将教师的服务工作界定为: 进行能使组织 (学校、学院、系所) 收益的 活动; 在学校各个领域提供领导工作; 促进
学校和社会联系, 包括实践和职业工作。同 样是从活动参与度和活动质量与影响力方 面, 对服务活动金考核, 建立了20项评估指 标体系，具体的指标如表4所示。

表4 昆士兰大学的服务工作评估指标

\begin{tabular}{|c|c|}
\hline 活动参与度指标 & 活动质量和影响力指标 \\
\hline 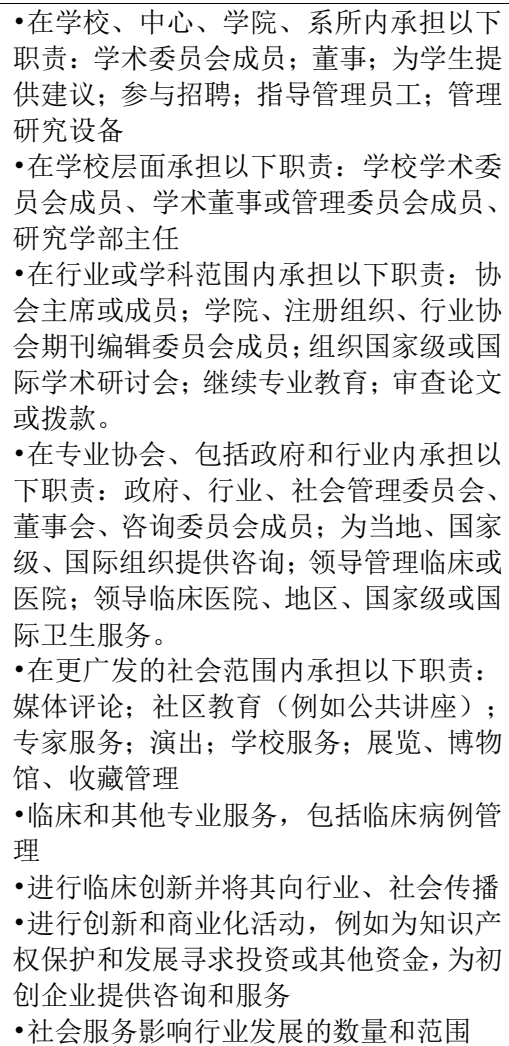 & $\begin{array}{l}\text {-影响内部或外部组织 } \\
\text { 政策 } \\
\text { •接受社会、行业机构、 } \\
\text { 重大媒体评论的专家咨 } \\
\text { 询建议邀请 } \\
\text {-对其他临床人员提供 } \\
\text { 专家建议, 为法律案件 } \\
\text { 提供技术指导和帮助 } \\
\text {-在经过审查和编辑的 } \\
\text { 出版物中有稳定地位 } \\
\text {-在经过审查的资助机 } \\
\text { 构中有稳定地位, 例如 } \\
\text { ARC 专家协会 } \\
\text {-领导地方、国家级、国 } \\
\text { 际的专业机构 } \\
\text { •因服务获得的任何水 } \\
\text { 平的奖励荣誉 } \\
\text {-领导和创新开发新临 } \\
\text { 床方法技术和职业化应 } \\
\text { 用 } \\
\text { •领导知识产权创业和 } \\
\text { 商业化发展, 并产生商 } \\
\text { 业化成果 } \\
\text { •有效参与临床医疗服 } \\
\text { 务 } \\
\text { •领导建立新的临床项 } \\
\text { 目, 管理相关临床团队 }\end{array}$ \\
\hline
\end{tabular}

\section{4考核指标细则}

针对教学研究岗、偏重教学岗、纯粹研 究岗、应用研究岗的每一个级别 (从A到E), 昆士兰大学都从研究、教学、服务三方面设 置了具体的考核要求, 不同类别的教师在具 体的考核指标方面会有不同的偏重。由于篇 幅的限制, 本文仅选取了每个岗位的C级作 为案例, 对昆士兰大学多通道的教师学术考 核指标进行介绍。 
表5 四个岗位C级的具体要求

\begin{tabular}{|c|c|}
\hline $\begin{array}{l}\text { 活动参与 } \\
\text { 度指标 }\end{array}$ & 活动质量指标 \\
\hline $\begin{array}{l}\text { 教学研究 } \\
\text { 岗 }\end{array}$ & 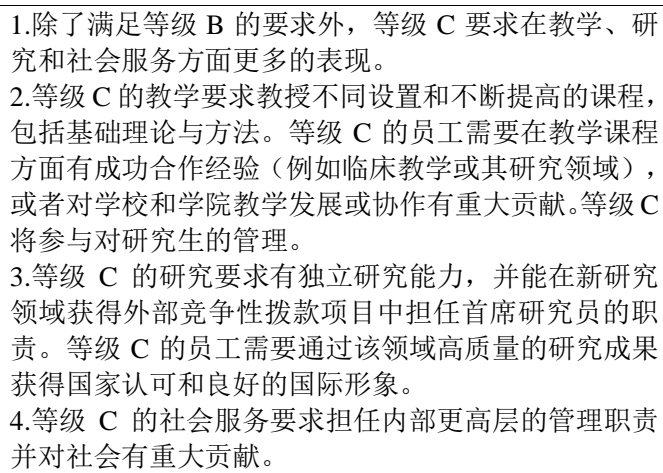 \\
\hline $\begin{array}{l}\text { 偏重教学 } \\
\text { 岗 }\end{array}$ & 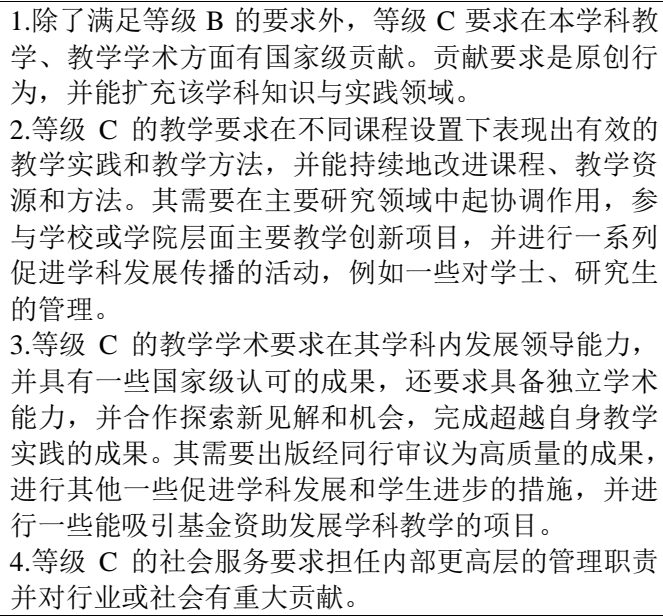 \\
\hline $\begin{array}{l}\text { 纯粹研究 } \\
\text { 岗 }\end{array}$ & $\begin{array}{l}\text { 1.除了满足等级 } \mathrm{B} \text { 的要求外, 等级 } \mathrm{C} \text { 要求在独立研究 } \\
\text { 中取得一定的成果。 } \\
\text { 2.等级 } \mathrm{C} \text { 的研究要求能独立进行研究, 在新领域机会 } \\
\text { 的探索研究中承担首席研究员的职责, 具有研究包括 } \\
\text { 研究培训与管理的领导能力, 能成功管理外部竞争性 } \\
\text { 拨款项目。其要求通过高质量成果获得国家级认可, } \\
\text { 并在国际范围内取得影响。 } \\
\text { 3. 等级 } \mathrm{C} \text { 要求较大程度地参与研究生管理。 } \\
\text { 4.承担教学职责时, 等级 } \mathrm{C} \text { 的教学需符合调查结果与 生学习成果的质量要求, 并应基于有效的反馈进行 } \\
\text { 改进和创新。 } \\
\text { 5. 等级 } \mathrm{C} \text { 的社会服务要求担任内部更高层的管理职责 } \\
\text { 并对行业或社会有重大贡献。 }\end{array}$ \\
\hline $\begin{array}{l}\text { 应用研究 } \\
\text { 岗 }\end{array}$ & $\begin{array}{l}\text { 1. 除了满足等级 B 的要求外, 等级 C 要求在临床教学、 } \\
\text { 研究、职业工作方面有出版的成果, 其中包括临床创 } \\
\text { 新。 } \\
\text { 2. 等级 } \mathrm{C} \text { 教学要求更大的成果, 例如能在临床教学课 } \\
\text { 程或专业课程中独当一面。其要求对更高级学生 (不 } \\
\text { 一定是研究生) 和专业培训项目进行管理。 } \\
\text { 3.等级 C 的临床研究要求出版同行审议的临床研究和 } \\
\text { 州或国家级临床实践的成果, 成功申请竞争性拨款项 } \\
\text { 目, 获得一个学术奖励或专业领域认可的奖励。更高 } \\
\text { 的专业资格认证和更高水平的研究同样被要求。 } \\
\text { 4. 等级 C 的社会服务要求获得职业水平上的领导能力, } \\
\text { 参与该领域专家协会。 }\end{array}$ \\
\hline
\end{tabular}

从表 5 可以看到, 昆士兰大学在指标的 设定上更多的是偏向于定性考核, 针对工作 职责作一个描述, 具体的评价依据教师的表 现而定, 这样就比较看重同行间的评议。

\section{3. 国内高校探索}

2014年，我国出台了《事业单位人事管 理条例》, 指出要明确岗位类别和等级, 同 时岗位应当具有明确的名称、职责任务、工 作标准和任职条件。《国家中长期教育改革 和发展规划纲要（2010-2020年）》也提出 要完善中国特色现代大学制度, 全面实行聘 任制度和岗位管理制度。这意味着我国高校 将面临重大的人事制度改革。从2010年起, 浙江、北京、上海、重庆、江苏、湖北等地 陆续修订职称申报政策, 允许教师高级职称 按照分类办法申报, 开展教师分类管理的相 关工作。浙江大学是全国高校中较早开展人 事制度改革的高校之一, 其中管理学院又是 浙江大学改革的院系试点, 因此本文将以浙 江大学管理学院为例, 简单介绍其进行教师 分类制度改革的情况。

在教师分类框架上, 浙江大学管理学院 同样存在横向和纵向两个结构。在横向上, 将教师分为了教学科学研究岗位 (Academic Track, 以下简称 $\mathrm{A}$ 岗) 、教学应用研究岗位

(Teaching Track, 以下简称T岗) 和咨询服 务岗位 (Professional Track, 以下简称P岗) 三种类型。A岗偏重基础研究、科学学位与 专业学位教学工作; T岗偏重应用研究、专 业学位与科学学位教学及研究、企业家培训 和企业咨询; P岗主要承担专业化管理服务、 企业咨询和教学等工作。在纵向上, 每种横 向岗位种类上都有数目不等的等级划分：A 岗一共有7级, 1 到5级及首聘岗和平台岗; $\mathrm{T}$ 岗一共有7级, 1到7级; $\mathrm{P}$ 岗一共有9级, 1 到 8 级及平台岗。

在学术活动分类和评价方面, 浙江大学 管理学院也将其分为了研究、教学、社会服 务三类进行相应的评价。研究工作分为为项 目和成果两方面, 项目包括主持国家级、省 部级和重大横向等项目的类别、数量及经费 等, 成果包括学术论著质量的要求、获奖情 况、专利及转化要求等, 具体以政府或学校 行政认定为判断依据。教学工作并不明确包 含教学学术这一概念, 而是单纯指对学生的 教育工作: 包括基本课程教学任务、教学质 量; 学生指导及思想政治教育工作等。社会 服务工作主要指院系的公共事务。 
在指标考核方面, 浙江大学管理学院偏 向于定量考核, 教师达到考核目标必须完成 最低的数值要求, 但给了教师选择自身工作 偏重的空间, 具体采用的是更具有弹性的 “基本指标 $+\mathrm{X}+\mathrm{Y}$ ” 模式, 其中, “基本考 核指标” 分为科研基本考核指标和教学基本 考核指标, 是聘期内应该完成的基本工作目 标, “ $X$ ” 为教学工作中的指标组合方案; “ $Y$ ” 为学科建设指标组合方案。

\section{4. 结束语}

通过对比分析, 我们发现昆士兰大学与 浙江大学管理学院在教师分类上具有较强 的相似性, 都是采用横纵结合的教师分类框 架, 从研究、教学和服务三个方面对教师活 动进行考核, 不过在具体工作指标的设定上 则具有一定的差异性, 主要体现为:

1. 昆士兰大学更为偏向定性考核, 浙江 大学管理学院更偏向定量考核。昆士兰大学 的指标是宽泛全面的, 这样就给教师更多自 由发挥的空间, 鼓励学术行为的多样性, 对 一些并非主流的研究领域予以支持。浙江大 学管理学院的指标更多的是可以精确衡量 的, 这样就降低了对教师工作考核中的主观 成分, 更具公平公正性。

2. 昆士兰大学兼顾教师工作过程与结 果, 浙江大学管理学院更注重于工作结果。 在考核中, 昆士兰大学全面地关注参与活 动、活动质量、活动影响, 这样就鼓励了具 有冒险性质的学术探索及公益性活动的产 生, 给予教师充分的学术自由, 但也增加了 考评管理的成本与难度。浙江大学管理学院 的指标主要是以国家或学校认可的学术成 就为主, 这样就有很强的指向性, 鼓励教师 进行与学校发展方向一致的努力, 能在较短 时间内实现跨越式发展。

就学术评价制度而言, 我国学术界面临 着国际化的压力, 一方面不能一味地追随国 外管理模式, 要保持自身发展的特点, 一方 面也要努力突破发展瓶颈, 克服当前种种不 足之处, 学习先进管理经验。因此我们认为 在制度设置上需遵从以下几个原则: 1 . 有用 性, 评价不能泛泛而谈, 而要有明确的目的 性, 能促进学校和教师的共同发展; 2. 可操
作性, 无论以定性评价为主还是以定量评价 为主, 都应简便易行; 3. 在公平公正的基础 上, 充分保障教师的学术自由; 4. 全面性, 应包含所有富有建设性的学术努力, 不仅仅 重视显性的学术成就, 也应包括隐性的学术 创造。

\section{References}

[1] J. Gu, and X. Dong, Academic Reflection and Assessment in American Higher Schools, Journal of Higher Education, Vol.23, pp. 100-104, 2002.

[2] C. Li, Perspectives on Deficiency and Correction of Academic Assessment in Universities and Colleges, Evaluation \& Management, Vol.5, pp. 19-23, 2007.

[3] T. Zhang, The Academic Evaluation Systems of University under The Perspective of Asymmetric Information Theory, Jiangsu Higher Education, Vol.5, pp. 46-48, 2010.

[4] E. L. Boyer, Scholarship Reconsidered: Priorities of the Professoriate. Princeton, N.J.: Carnegie Foundation for the Advancement of Teaching, 1990.

[5] E. F. Martin, and L. M. James, Evidence-based Appointment and Promotion of Academic Faculty at the University of Chicago, Academic Medicine, Vol.83, pp. 85-95, 2008.

[6] S. Ahmady, T. Changiz, M. Brommels, F. A. Gaffney, J. Thor, and I. Masiello, Contextual adaptation of the Personnel Evaluation Standards for assessing faculty evaluation systems in developing countries: the case of Iran, BMC Medical Education, Vol.9, pp.9-18, 2009.

[7] D. R. Hong, A critical study on the university and academic assessment system in Korea, Inter-Asia Cultural Studies, Vol.10, pp. 292302, 2009.

[8] H. Patricia, C. Michelle, Evaluating faculty work: expectations and standards of faculty performance in research universities, Research Papers in Education, Vol.24, pp. 383-3419, 2009. 